\title{
On centro-affine curves and Bäcklund transformations of the $\mathrm{KdV}$ equation
}

\author{
Serge Tabachnikov*
}

\section{A family of transformations on the space of curves}

This note stems from [1] where we study the integrable dynamics of a 1parameter family of correspondences on ideal polygons in the hyperbolic plane and hyperbolic space: two $n$-gons $P=\left(p_{1}, p_{2}, \ldots\right)$ and $Q=\left(q_{1}, q_{2}, \ldots\right)$ in $\mathbb{R P}^{1}$ or in $\mathbb{C P}^{1}$ are in correspondence $P \stackrel{c}{\sim} Q$ if $\left[p_{i}, p_{i+1}, q_{i}, q_{i+1}\right]=c$ for all $i$; the constant $c$ is a parameter.

In the limit $n \rightarrow \infty$, a polygon becomes a parameterized curve. The ground field can be either $\mathbb{R}$ of $\mathbb{C}$; to fix ideas, choose $\mathbb{R}$. Let us use the following definition of cross-ratio to define our correspondence (other five definitions result in the change of the constant $c)$ :

$$
\left[p_{i}, p_{i+1}, q_{i}, q_{i+1}\right]=\frac{\left(q_{i+1}-q_{i}\right)\left(p_{i+1}-p_{i}\right)}{\left(q_{i}-p_{i}\right)\left(q_{i+1}-p_{i+1}\right)}=c .
$$

We replace polygons by non-degenerate closed curves $\gamma: \mathbb{R} \rightarrow \mathbb{R P}^{1}$ with $\gamma^{\prime}(t)>0$; to be concrete, let the period be $\pi: \gamma(t+\pi)=\gamma(t)$. Also let us assume that the rotation number of the curve $\gamma$ is 1 , that is, $\gamma: \mathbb{R} / \pi \mathbb{Z} \rightarrow$ $\mathbb{R} \mathbb{P}^{1}$ is a diffeomorphism. Denote the space of such curves by $\widetilde{\mathcal{C}}$ and let $\mathcal{C}=\widetilde{\mathcal{C}} / \operatorname{PSL}(2, \mathbb{R})$ be the moduli space.

Then a continuous version of (11) is

$$
\frac{\gamma^{\prime}(t) \delta^{\prime}(t)}{(\delta(t)-\gamma(t))^{2}}=c .
$$

*Department of Mathematics, Penn State University, University Park, PA 16802; tabachni@math.psu.edu 
Write $\gamma \stackrel{c}{\sim} \delta$ to denote this relation on $\widetilde{\mathcal{C}}$. Since cross-ratio is Möbius invariant, we also have a relation on $\mathcal{C}$ which we continue to denote by $\stackrel{c}{\sim}$. Note that $\stackrel{c}{\sim}$ is a symmetric relation.

Lemma 1.1 For a generic curve $\gamma$, the relation $\gamma \stackrel{c}{\sim} \delta$ is a (partially defined) 2-2 $\operatorname{map} T_{c}: \gamma \mapsto \delta$.

Proof. Given $\gamma(t)$, equation (2) is a Riccati equation on $\delta(t)$, its monodromy is a Möbius transformation (see, e.g., [5]) which has either two or no fixed points, unless it is the identity. Over $\mathbb{C}$, there are always two fixed points (possibly, coinciding), and over $\mathbb{R}$, we need to assume that they exist. Then $\stackrel{c}{\sim}$ defines a $2-2$ map.

Thus, given $\gamma$, there are two choices of $\delta=T_{c}(\gamma)$. Once a choice is made, one similarly has two choices for $T_{c}(\delta)$, but one of them is $\gamma$, so we choose the other one, and so on. Hence the choice of $\delta$ determines the map $T_{c}$; the other choice gives the inverse map $T_{c}^{-1}$.

Following the standard procedure (see, e.g., [7]), lift a curve $\gamma(t)$ from $\mathbb{R} \mathbb{P}^{1}$ to $\mathbb{R}^{2}$, normalizing the lift $\Gamma(t)$ so that $\left[\Gamma, \Gamma^{\prime}\right]=1$ (here and elsewhere $[$,$] denotes the determinant made by two vectors).$

Explicitly, $\Gamma=\left(\left(\gamma^{\prime}\right)^{-1 / 2},\left(\gamma^{\prime}\right)^{-1 / 2} \gamma\right)$. Note the square root: the curve $-\Gamma$ will do as well, the lift is defined up to the sign, and the action of $\operatorname{PSL}(2, \mathbb{R})$ is replaced by that of $\operatorname{SL}(2, \mathbb{R})$. We obtain centro-affine realizations of the spaces $\widetilde{\mathcal{C}}$ and $\mathcal{C}$.

The curve $\Gamma$ satisfies a Hill equation

$$
\Gamma^{\prime \prime}(t)=p(t) \Gamma(t)
$$

with a $\pi$-periodic potential $p(t)$, and $\Gamma(t+\pi)=-\Gamma(t)$ (the curve makes exactly half-rotation on $[0, \pi])$. In geometric terms, the potential $p$ is the (negative) centro-affine curvature of the curve $\Gamma$.

In these terms, equation (1) becomes

$$
\frac{[\Delta(t), \Gamma(t)][\Delta(t+\varepsilon), \Gamma(t+\varepsilon)]}{[\Delta(t), \Delta(t+\varepsilon)][\Gamma(t), \Gamma(t+\varepsilon)]}=\mathrm{const},
$$

and, in the limit $\varepsilon \rightarrow 0$, we obtain an analog of equation (2):

$$
[\Gamma(t), \Delta(t)]^{2}=c^{2}
$$


Break the symmetry between $\Gamma$ and $\Delta$ by taking square root:

$$
[\Gamma(t), \Delta(t)]=c .
$$

This defines a map on the lifted curves: $T_{c}(\Gamma)=\Delta$. Note that $T_{c}(\Delta)=-\Gamma$.

Lemma 1.2 $T_{c}: \Gamma \mapsto \Delta$ is a (partially defined) 2-2 map.

Proof. Let us search for $\Delta$ in the form $\Delta=a \Gamma+b \Gamma^{\prime}$, where $a$ and $b$ are $\pi$-periodic functions. Then equation $[\Gamma, \Delta]=c$ implies that $b(t)=c$, $\Delta=a \Gamma+c \Gamma^{\prime}$, and then $\Delta^{\prime}=\left(a^{\prime}+c p\right) \Gamma+a \Gamma^{\prime}$. The condition $\left[\Delta, \Delta^{\prime}\right]=1$ now implies

$$
a^{\prime}=\frac{a^{2}-1}{c}-c p .
$$

This is a Riccati equation on function $a(t)$ with periodic coefficients. The monodromy of this equation is a Möbius transformation, hence it has two fixed points (always, if one works over $\mathbb{C}$, and over $\mathbb{R}$ one needs to assume that it does), corresponding to two periodic solutions of (5). Each solution defines a curve $\Delta$ with $T_{c}(\Gamma)=\Delta$.

As before, once a choice of a fixed point of the monodromy is made, the map becomes 1-1: of the two choices available for the next curve $\Delta$, one is extraneous because it takes one back to $-\Gamma$.

\section{Two pre-symplectic forms and a bi-Hamiltonian structure}

Starting with U. Pinkall [9, a number of recent papers were devoted to the study of the Korteweg-de Vries equation in terms of cento-affine curves [2, 3, 4, 11]. Let us present the relevant results.

A tangent vector at a cento-affine curve $\Gamma$ is a vector field along $\Gamma$ that can be written as a linear combination $h \Gamma+f \Gamma^{\prime}$ where $h, f$ are $\pi$-periodic functions.

Lemma 2.1 The function $f$ is arbitrary, and $h=-\frac{1}{2} f^{\prime}$. 
Proof. If $\Gamma+\varepsilon v$ is a deformation of $\Gamma$, then $\left[\Gamma, v^{\prime}\right]=\left[\Gamma^{\prime}, v\right]$ because $\left[\Gamma, \Gamma^{\prime}\right]=$ 1. For $v=h \Gamma+f \Gamma^{\prime}$, this implies that $h=-\frac{1}{2} f^{\prime}$.

Denote the tangent vectors by $U, V$ or $U_{f}, V_{f}$, in the format $-\frac{1}{2} f^{\prime} \Gamma+f \Gamma^{\prime}$. The following pre-symplectic structure on space $\widetilde{\mathcal{C}}$ was introduced in $[9$. Let $U, V$ be tangent vector fields along $\Gamma$; define

$$
\omega(U, V)=\int_{\Gamma}[U, V] d t
$$

that is,

$$
\omega\left(U_{f}, V_{g}\right)=\frac{1}{2} \int_{0}^{\pi}\left(f g^{\prime}-f^{\prime} g\right) d t .
$$

The kernel of $\omega$ is spanned by the field $\Gamma^{\prime}$, that is, by the reparameterizations $t \mapsto t+$ const.

Pinkall observed that the Hamiltonian vector field of the function $\int p d t$ is $U_{p}$, which induces the $\mathrm{KdV}$ evolution of the potential $p$

$$
\dot{p}=-\frac{1}{2} p^{\prime \prime \prime}+3 p^{\prime} p
$$

(the signs differ from those of Pinkall because he used the opposite sign for the potential of Hill's equation).

The second pre-symplectic structure was introduced in [4]: for tangent vector fields $U, V$ along $\Gamma$, let

$$
\Omega(U, V)=\int_{\Gamma}\left(\left[U^{\prime}, V^{\prime}\right]+p[U, V]\right) d t
$$

that is,

$$
\Omega\left(U_{f}, V_{g}\right)=\int_{0}^{\pi}\left[\frac{1}{4}\left(f^{\prime} g^{\prime \prime}-f^{\prime \prime} g^{\prime}\right)+p\left(f g^{\prime}-f^{\prime} g\right)\right] d t .
$$

Concerning the kernel of $\Omega$, one has

Lemma 2.2 ([4]) The kernel of $\Omega$ is 3-dimensional, it is generated by the Killing vector fields $A(\Gamma)$ with $A \in \mathrm{SL}(2)$. 
Proof. One has

$$
\Omega(U, V)=\int\left[p U-U^{\prime \prime}, V\right] d t .
$$

Hence $U$ is in the kernel if and only if $U^{\prime \prime}=p U$, that is, $U(t)$ is $\operatorname{SL}(2)$ equivalent to $\Gamma(t)$.

Thus the form $\Omega$ descends on the moduli space $\mathcal{C}$ as a symplectic form.

It is shown in [2, 11, 4] that the forms $\omega$ and $\Omega$ provide a bi-Hamiltonian structure on $\widetilde{\mathcal{C}}$, corresponding to a pair of compatible Poisson brackets for the $\mathrm{KdV}$ equation.

Namely, let $X_{0}, X_{1}, \ldots$ and $H_{1}, H_{2}, \ldots$ be the vector fields and the Hamiltonians of the KdV hierarchy in terms of centro-affine curves:

$$
X_{0}=U_{1}=\Gamma^{\prime}, X_{1}=U_{p}=-\frac{p^{\prime}}{2} \Gamma+p \Gamma^{\prime}, \ldots, H_{1}=\int p d t, H_{2}=\frac{1}{2} \int p^{2} d t, \ldots
$$

Then one has

$$
\Omega\left(X_{j-1}, \cdot\right)=d H_{j}=\omega\left(X_{j}, \cdot\right), j=1,2, \ldots
$$

see [4].

The forms $\omega$ and $\Omega$ on projective curves. Let us calculate these forms in terms of the curves $\gamma: \mathbb{R} \rightarrow \mathbb{R P}^{1}$.

In [1], the following differential 2-form on the space of polygons $\left(p_{1}, \ldots, p_{n}\right) \subset$ $\mathbb{R P}^{1}$ was considered

$$
\omega^{\prime}=\sum_{i} \frac{d p_{i} \wedge d p_{i+1}}{\left(p_{i+1}-p_{i}\right)^{2}},
$$

and it was proved that this form was $T_{c}$-invariant. In the continuous limit, a polygon becomes a curve $\gamma(t)$. Let $u(t), v(t)$ be two vector fields along $\gamma(t)$, that is, two periodic functions. Then, in the continuous limit, we obtain the form

$$
\omega^{\prime}(u, v)=\int \frac{u v^{\prime}-u^{\prime} v}{\left(\gamma^{\prime}\right)^{2}} d t .
$$

Lemma 2.3 One has $\omega=\frac{1}{2} \omega^{\prime}$. 
Proof. Since

$$
\Gamma_{1}=\left(\gamma^{\prime}\right)^{-1 / 2}, \Gamma_{2}=\left(\gamma^{\prime}\right)^{-1 / 2} \gamma
$$

one calculates the respective vector field along $\Gamma$ :

$$
U=\left(-\frac{1}{2} u^{\prime} \Gamma_{1}^{3},-\frac{1}{2} u^{\prime} \Gamma_{1}^{2} \Gamma_{2}+u \Gamma_{1}\right)
$$

and likewise for $V$. Then

$$
[U, V]=\frac{1}{2} \Gamma_{1}^{4}\left(u v^{\prime}-u^{\prime} v\right)
$$

and the result follows.

By Lemma 2.2, the 2-form $\Omega$ descends to the moduli space of projective curves, that is, to the space of Hill's equations. This space is a coadjoint orbit of the Virasoro algebra, and $\Omega$ coincides (up to a factor) with the celebrated Kirillov-Kostant-Souriau symplectic structure, see, e.g., [6, 7] for this material.

Namely, let $\gamma$ be a curve in $\mathbb{R P}^{1}$, and let $u$ and $v$ be vector fields along $\gamma$. The Kirillov-Kostant-Souriau symplectic form is given by the formula

$$
\Omega^{\prime}(u, v)=\int \frac{u^{\prime \prime}(t) v^{\prime}(t)-u^{\prime}(t) v^{\prime \prime}(t)}{\gamma^{\prime}(t)^{2}} d t
$$

see, e.g., [8].

Lemma 2.4 One has

$$
\Omega=-\frac{1}{4} \Omega^{\prime}
$$

Proof. As in the proof of Lemma 2.3.

$$
U=\left(-\frac{1}{2} u^{\prime} \Gamma_{1}^{3},-\frac{1}{2} u^{\prime} \Gamma_{1}^{2} \Gamma_{2}+u \Gamma_{1}\right)
$$

and then

$U^{\prime}=\left(-\frac{1}{2} u^{\prime \prime} \Gamma_{1}^{3}-\frac{3}{2} u^{\prime} \Gamma_{1}^{2} \Gamma_{1}^{\prime},-\frac{1}{2} u^{\prime \prime} \Gamma_{1}^{2} \Gamma_{2}-u^{\prime} \Gamma_{1} \Gamma_{2} \Gamma_{1}^{\prime}-\frac{1}{2} u^{\prime} \Gamma_{1}^{2} \Gamma_{2}^{\prime}+u^{\prime} \Gamma_{1}+u \Gamma_{1}^{\prime}\right)$.

Similar formulas hold for $V$. 
Now one computes, using the fact that $\Gamma_{2}^{\prime} \Gamma_{1}-\Gamma_{1}^{\prime} \Gamma_{2}=1$,

$$
\left[U^{\prime}, V^{\prime}\right]=-\frac{1}{4} \Gamma_{1}^{4}\left(u^{\prime \prime} v^{\prime}-u^{\prime} v^{\prime \prime}\right)-\frac{1}{2} \Gamma_{1}^{3} \Gamma_{1}^{\prime}\left(u^{\prime \prime} v-u v^{\prime \prime}\right)-\frac{3}{2} \Gamma_{1}^{2}\left(\Gamma_{1}^{\prime}\right)^{2}\left(u^{\prime} v-u v^{\prime}\right),
$$

and

$$
p[U, V]=-\frac{1}{2} p \Gamma_{1}^{4}\left(u^{\prime} v-u v^{\prime}\right)=-\frac{1}{2} \Gamma_{1}^{3} \Gamma_{1}^{\prime \prime}\left(u^{\prime} v-u v^{\prime}\right) .
$$

Integrating by parts,

$$
-\int \Gamma_{1}^{3} \Gamma_{1}^{\prime}\left(u^{\prime \prime} v-u v^{\prime \prime}\right) d t=\int\left(\Gamma_{1}^{3} \Gamma_{1}^{\prime \prime}+3 \Gamma_{1}^{2}\left(\Gamma_{1}^{\prime}\right)^{2}\right)\left(u^{\prime} v-u v^{\prime}\right) d t
$$

and collecting terms,

$\Omega(U, V)=\int\left(\left[U^{\prime}, V^{\prime}\right]+p[U, V]\right) d t=-\frac{1}{4} \int \Gamma_{1}^{4}\left(u^{\prime \prime} v^{\prime}-u^{\prime} v^{\prime \prime}\right) d t=-\frac{1}{4} \Omega^{\prime}(u, v)$, as claimed.

\section{$3 \quad T_{c}$-invariance of the bi-Hamiltonian struc- ture and complete integrability of the trans- formations $T_{c}$}

Let $T_{c}(\Gamma)=\Delta$ with $\Delta^{\prime \prime}=q \Delta$; one can write $\Delta(t)=a(t) \Gamma(t)+c \Gamma^{\prime}(t)$, where $a(t)$ is a periodic function.

Lemma 3.1 One has:

$$
\Gamma=a \Delta-c \Delta^{\prime}, p+q=\frac{2}{c^{2}}\left(a^{2}-1\right), q-p=\frac{2}{c} a^{\prime} .
$$

Proof. Since $[\Delta,-\Gamma]=c$, we can write $-\Gamma=b \Delta+c \Delta^{\prime}$ where $b(t)$ is a periodic function. Substitute $\Delta=a \Gamma+c \Gamma^{\prime}$ in this equation to find that $b=-a$. We also have an analog of (5) for function $b(t): c b^{\prime}=b^{2}-1-c^{2} q$. This implies the relations between $p$ and $q$ stated in the lemma.

Let $T_{c}(\Gamma)=\Delta$, and let $U_{f}, V_{g}$ be two tangent vectors, at $\Gamma$ and $\Delta$, respectively, related by the differential of $T_{c}$.

Lemma 3.2 One has

$$
\frac{c}{2}\left(f^{\prime}+g^{\prime}\right)=a(g-f),
$$

where the function $a(t)$ is as above. 
Proof. One has $[U, \Delta]+[\Gamma, V]=0$, or

$$
\frac{c}{2}\left(f^{\prime}+g^{\prime}\right)=f\left[\Gamma^{\prime}, \Delta\right]+g\left[\Gamma, \Delta^{\prime}\right]=a(g-f),
$$

where the last equality makes use of $\Delta=a \Gamma+c \Gamma^{\prime}$ and of $\left[\Gamma^{\prime}, \Delta\right]+\left[\Gamma, \Delta^{\prime}\right]=0$.

The following theorem is our main observation.

Theorem 1 The forms $\omega$ and $\Omega$ are invariant under the maps $T_{c}$ :

$$
T_{c}^{*}(\omega)=\omega, T_{c}^{*}(\Omega)=\Omega .
$$

Proof. Let $T_{c}(\Gamma)=\Delta$, and let $U_{f_{i}}, V_{g_{i}}, i=1,2$, be two pairs of tangent vectors, at $\Gamma$ and $\Delta$, respectively, related by the differential of $T_{c}$. One has

$$
\begin{array}{r}
\int\left[\left(g_{1}^{\prime} g_{2}-g_{1} g_{2}^{\prime}\right)-\left(f_{1}^{\prime} f_{2}-f_{1} f_{2}^{\prime}\right)\right] d t=\int\left[\left(g_{1}^{\prime} g_{2}-g_{1} g_{2}^{\prime}\right)-\left(f_{1}^{\prime} f_{2}-f_{1} f_{2}^{\prime}\right)\right. \\
\left.-\left(g_{1}^{\prime} f_{2}+g_{1} f_{2}^{\prime}\right)+\left(g_{2}^{\prime} f_{1}+g_{2} f_{1}^{\prime}\right)\right] d t \\
=\int\left[\left(f_{1}^{\prime}+g_{1}^{\prime}\right)\left(g_{2}-f_{2}\right)-\left(f_{2}^{\prime}+g_{2}^{\prime}\right)\left(g_{1}-f_{1}\right)\right] d t=0,
\end{array}
$$

where the first equality follows from the fact that $g_{1}^{\prime} f_{2}+g_{1} f_{2}^{\prime}=\left(g_{1} f_{2}\right)^{\prime}$ and $g_{2}^{\prime} f_{1}+g_{2} f_{1}^{\prime}=\left(g_{2} f_{1}\right)^{\prime}$, which integrates to zero, and the last equality follows from (77). Thus $T_{c}^{*}(\omega)=\omega$.

To prove that $T_{c}^{*}(\Omega)=\Omega$, we argue similarly, although the computation is more involved.

Differentiate (7) to obtain

$$
\frac{c}{2}\left(f^{\prime \prime}+g^{\prime \prime}\right)=a^{\prime}(g-f)+a\left(g^{\prime}-f^{\prime}\right) .
$$

We want to show that the integral

$$
\int\left(\frac{1}{4}\left(f_{1}^{\prime} f_{2}^{\prime \prime}-f_{1}^{\prime \prime} f_{2}^{\prime}\right)+p\left(f_{1} f_{2}^{\prime}-f_{1}^{\prime} f_{2}\right)-\frac{1}{4}\left(g_{1}^{\prime} g_{2}^{\prime \prime}-g_{1}^{\prime \prime} g_{2}^{\prime}\right)-q\left(g_{1} g_{2}^{\prime}-g_{1}^{\prime} g_{2}\right)\right) d t
$$

vanishes. One has

$f_{1}^{\prime} f_{2}^{\prime \prime}-f_{1}^{\prime \prime} f_{2}^{\prime}-g_{1}^{\prime} g_{2}^{\prime \prime}+g_{1}^{\prime \prime} g_{2}^{\prime}=\left(f_{1}^{\prime \prime}+g_{1}^{\prime \prime}\right)\left(g_{2}^{\prime}-f_{2}^{\prime}\right)-\left(f_{2}^{\prime \prime}+g_{2}^{\prime \prime}\right)\left(g_{1}^{\prime}-f_{1}^{\prime}\right)+\left(f_{2}^{\prime} g_{1}^{\prime}-f_{1}^{\prime} g_{2}^{\prime}\right)^{\prime}$, 
hence

$$
\begin{aligned}
& \frac{1}{4} \int\left(f_{1}^{\prime} f_{2}^{\prime \prime}-f_{1}^{\prime \prime} f_{2}^{\prime}-g_{1}^{\prime} g_{2}^{\prime \prime}+g_{1}^{\prime \prime} g_{2}^{\prime}\right) d t \\
= & \frac{1}{2 c} \int\left\{\left[a^{\prime}\left(g_{1}-f_{1}\right)+a\left(g_{1}^{\prime}-f_{1}^{\prime}\right)\right]\left(g_{2}^{\prime}-f_{2}^{\prime}\right)-\left[a^{\prime}\left(g_{2}-f_{2}\right)+a\left(g_{2}^{\prime}-f_{2}^{\prime}\right)\right]\left(g_{1}^{\prime}-f_{1}^{\prime}\right)\right\} d t \\
= & \int \frac{a^{\prime}}{2 c}\left[\left(g_{1}-f_{1}\right)\left(g_{2}^{\prime}-f_{2}^{\prime}\right)-\left(g_{2}-f_{2}\right)\left(g_{1}^{\prime}-f_{1}^{\prime}\right)\right] d t,
\end{aligned}
$$

where the first equality follows from (8).

Next we evaluate the remaining part of the integral (9), using Lemma 3.1 .

$$
\begin{aligned}
& \int\left[p\left(f_{1} f_{2}^{\prime}-f_{1}^{\prime} f_{2}\right)-q\left(g_{1} g_{2}^{\prime}-g_{1}^{\prime} g_{2}\right)\right] d t \\
= & \int \frac{a^{2}-1}{c^{2}}\left(f_{1} f_{2}^{\prime}-f_{1}^{\prime} f_{2}-g_{1} g_{2}^{\prime}+g_{1}^{\prime} g_{2}\right) d t-\int \frac{a^{\prime}}{c}\left(f_{1} f_{2}-f_{1}^{\prime} f_{2}+g_{1} g_{2}^{\prime}-g_{1}^{\prime} g_{2}\right) d t .
\end{aligned}
$$

Collecting the integrals together, we obtain

$$
\begin{aligned}
& \int \frac{a^{\prime}}{2 c}\left[\left(f_{1}^{\prime}+g_{1}^{\prime}\right)\left(f_{2}+g_{2}\right)-\left(f_{2}^{\prime}+g_{2}^{\prime}\right)\left(f_{1}+g_{1}\right)\right] d t \\
+ & \int \frac{a^{2}-1}{c^{2}}\left(f_{1} f_{2}^{\prime}-f_{1}^{\prime} f_{2}-g_{1} g_{2}^{\prime}+g_{1}^{\prime} g_{2}\right) d t \\
= & \int \frac{2 a a^{\prime}}{c^{2}}\left(f_{2} g_{1}-f_{1} g_{2}\right) d t+\int \frac{a^{2}-1}{c^{2}}\left(f_{1} f_{2}^{\prime}-f_{1}^{\prime} f_{2}-g_{1} g_{2}^{\prime}+g_{1}^{\prime} g_{2}\right) d t
\end{aligned}
$$

where the equality is due to (7).

Finally, notice that $\left(a^{2}-1\right)^{\prime}=2 a a^{\prime}$, and integrate by parts to obtain

$$
\begin{array}{r}
\int \frac{a^{2}-1}{c^{2}}\left[\left(f_{1} f_{2}^{\prime}-f_{1}^{\prime} f_{2}-g_{1} g_{2}^{\prime}+g_{1}^{\prime} g_{2}\right)-\left(f_{2}^{\prime} g_{1}+f_{2} g_{1}^{\prime}-f_{1}^{\prime} g_{2}-f_{1} g_{2}^{\prime}\right)\right] d t \\
=\int \frac{a^{2}-1}{c^{2}}\left[\left(f_{2}^{\prime}+g_{2}^{\prime}\right)\left(f_{1}-g_{1}\right)-\left(f_{1}^{\prime}+g_{1}^{\prime}\right)\left(f_{2}-g_{2}\right)\right] d t=0
\end{array}
$$

since the last integrand vanishes due to (7).

Corollary 2 The maps $T_{c}$ commute with the $K d V$ flows and preserve the KdV integrals. 
Proof. One argues inductively using formulas (6):

$$
\Omega\left(X_{j-1}, \cdot\right)=d H_{j}=\omega\left(X_{j}, \cdot\right) .
$$

If $T_{c}$ preserves $X_{j-1}$ then, since it also preserves $\Omega$, it preserves $d H_{j}$. If $T_{c}$ preserves $d H_{j}$ then, since it preserves $\omega$, it also preserves $X_{j}$.

To start the induction, we check that $\int p d t$ is invariant:

$$
\int(q(t)-p(t)) d t=\frac{2}{c} \int a^{\prime}(t) d t=0
$$

due to Lemma 3.1

Since $d H_{j}$ is preserved, it could be that $T_{c}$ changes $H_{j}$ by a constant. To see that this constant is zero, let $\Gamma$ be the circle $(\cos t, \sin t)$. Then $\Delta$ differs from $\Gamma$ by a parameter shift, and the values of the functions $H_{j}$ on $\Gamma$ and $\Delta$ are equal.

Thus the transformations $T_{c}$ are symmetries of the Korteweg-de Vries equation.

Remark 3.3 The argument above is similar to the one given in [10] which concerned with the filament equation and the bicycle transformations as its symmetries.

Additional integrals. Let $\Gamma=\left(\Gamma_{1}, \Gamma_{2}\right)$. Consider the functions

$$
I=\int \Gamma_{1}^{2} d t, J=\int \Gamma_{1} \Gamma_{2} d t, K=\int \Gamma_{2}^{2} d t
$$

on the space of centro-affine curves.

Proposition 3.4 The functions $I, J, K$ are the Hamiltonians of the generator of the action of $\operatorname{sl}(2, \mathbb{R})$ on $\widetilde{\mathcal{C}}$ with respect to the D-form $\omega$. The function $I K-J^{2}$ is $\mathrm{SL}(2, \mathbb{R})$-invariant.

Proof. The generators of $\operatorname{sl}(2, \mathbb{R})$ are the fields

$$
\left(\Gamma_{2}, 0\right),\left(\Gamma_{1},-\Gamma_{2}\right),\left(0, \Gamma_{1}\right) .
$$

Let us consider the first one; the other ones are dealt with similarly. 
We claim that $\left(\Gamma_{2}, 0\right)=-V_{\Gamma_{2}^{2}}$. Indeed,

$$
V_{\Gamma_{2}^{2}}=-\Gamma_{2} \Gamma_{2}^{\prime} \Gamma+\Gamma_{2}^{2} \Gamma^{\prime}
$$

The first component of this vector is $-\Gamma_{2}\left(\Gamma_{2}^{\prime} \Gamma_{1}-\Gamma_{1}^{\prime} \Gamma_{2}\right)=-\Gamma_{2}$, and the second component is $-\Gamma_{2}^{\prime} \Gamma_{2}^{2}+\Gamma_{2}^{\prime} \Gamma_{2}^{2}=0$.

Let $U_{f}$ be a test vector field. Then

$$
d K\left(U_{f}\right)=\int \Gamma_{2}\left(\Gamma_{2}^{\prime} f-\frac{1}{2} \Gamma_{2} f^{\prime}\right) d t=2 \int \Gamma_{2} \Gamma_{2}^{\prime} f d t
$$

On the other hand,

$$
\omega\left(U_{f}, U_{\Gamma_{2}^{2}}\right)=\int 2 \Gamma_{2} \Gamma_{2}^{\prime} f d t
$$

as needed.

As to $\operatorname{sl}(2, \mathbb{R})$ invariance of $I K-J^{2}$, let us again check invariance under the field $\left(\Gamma_{2}, 0\right)$ (the rest is similar). Calculating mod $\varepsilon^{2}$, one has

$$
\begin{array}{r}
\left(\int\left(\Gamma_{1}+\varepsilon \Gamma_{2}\right)^{2} d t\right)\left(\int \Gamma_{2}^{2} d t\right)-\left(\int\left(\Gamma_{1}+\varepsilon \Gamma_{2}\right) \Gamma_{2} d t\right)^{2}=I K-J^{2} \\
+2 \varepsilon\left[\left(\int \Gamma_{1} \Gamma_{2} d t\right)\left(\int \Gamma_{2}^{2} d t\right)-\left(\int \Gamma_{1} \Gamma_{2} d t\right)\left(\int \Gamma_{2}^{2} d t\right)\right]=I K-J^{2},
\end{array}
$$

as needed

Next we show that $I, J, K$ are integrals of the transformations $T_{c}$.

Theorem 3 Let $T_{c}(\Gamma)=\Delta$, then

$$
I(\Gamma)=I(\Delta), J(\Gamma)=J(\Delta), K(\Gamma)=K(\Delta) .
$$

Proof. Consider the case of $I$; the other two cases are similar.

We have $\Delta=a \Gamma+c \Gamma^{\prime}$, and we want to show that $\int \Delta_{1}^{2}=\int \Gamma_{1}^{2}$. Indeed,

$$
\begin{aligned}
& \int\left(\Delta_{1}^{2}-\Gamma_{1}^{2}\right) d t=\int\left[\left(a^{2}-1\right) \Gamma_{1}^{2}+2 c a \Gamma_{1} \Gamma_{1}^{\prime}+c^{2}\left(\Gamma_{1}^{\prime}\right)^{2}\right] d t \\
& =\int\left[\left(a^{2}-1-c a^{\prime}\right) \Gamma_{1}^{2}+c^{2}\left(\Gamma_{1}^{\prime}\right)^{2}\right] d t=c^{2} \int\left[p \Gamma_{1}^{2}+\left(\Gamma_{1}^{\prime}\right)^{2}\right] d t \\
& =c^{2} \int\left[\Gamma_{1}^{\prime \prime} \Gamma_{1}+\left(\Gamma_{1}^{\prime}\right)^{2}\right] d t=0,
\end{aligned}
$$

where the second equality is integration by parts, the third is due to (5) , the fourth is due to $\Gamma^{\prime \prime}=p \Gamma$, and the last one is again integration by parts. 


\section{Monodromy integrals and permutability}

Now we describe an infinite collection of $\operatorname{SL}(2, \mathbb{R})$-invariant integrals of the maps $T_{c}$ that arise from the monodromy of the Riccati equations.

Let $x$ be an affine coordinate on $\mathbb{R P}^{1}$. The Lie algebra $\operatorname{sl}(2, \mathbb{R})$ is generated by the vector fields $\partial_{x}, x \partial_{x}, x^{2} \partial_{x}$. Introduce time-dependent vector fields, depending on $\gamma(t)$ or $\delta(t)$, respectively, taking values in $\operatorname{sl}(2, \mathbb{R})$ for each $t$ :

$$
\xi_{\gamma}=\left(\frac{\gamma^{2}}{\gamma^{\prime}}-2 \frac{\gamma}{\gamma^{\prime}} x+\frac{1}{\gamma^{\prime}} x^{2}\right) \partial_{x}, \quad \xi_{\delta}=\left(\frac{\delta^{2}}{\delta^{\prime}}-2 \frac{\delta}{\delta^{\prime}} x+\frac{1}{\delta^{\prime}} x^{2}\right) \partial_{x} .
$$

Then equation (2) describes $\delta$ as evolving under the field $c \xi_{\gamma}$ and, equivalently, $\gamma$ as evolving under $c \xi_{\delta}$.

Fix a (spectral) parameter $\lambda$, and consider the time- $\pi$ flows of the fields $\lambda \xi_{\gamma}$ and $\lambda \xi_{\delta}$, where $\gamma$ and $\delta$ are related by (2). Denote these projective transformations of $\mathbb{R P}^{1}$ by $\Phi_{\lambda, \gamma}$ and $\Phi_{\lambda, \delta}$.

Theorem 4 For every $\lambda$, the maps $\Phi_{\lambda, \gamma}$ and $\Phi_{\lambda, \delta}$ are conjugate in $\operatorname{PSL}(2, \mathbb{R})$.

It follows that the spectral invariants of $\Phi_{\lambda, \gamma}$, say $\operatorname{Tr}^{2} / \operatorname{det}$, as functions of $\lambda$, are integrals of the maps $T_{c}$ for all values of $c$.

Proof. Let $\gamma$ and $\delta$ satisfy (2). Introduce a time-dependent matrix, also depending on parameter $\mu$ :

$$
A_{\mu, \gamma, \delta}(t)=\frac{1}{\gamma(t)-\delta(t)}\left[\begin{array}{cc}
\gamma(t)-\mu \delta(t), & \gamma(t) \delta(t)(\mu-1) \\
1-\mu, & \gamma(t) \mu-\delta(t)
\end{array}\right] .
$$

We claim that if $\lambda=c(1-\mu)$, then $A_{\mu, \gamma, \delta}(t)$ conjugates the vector fields $\lambda \xi_{\gamma}$ and $\lambda \xi_{\delta}$.

Namely, let $\varepsilon$ be an infinitesimal parameter, and set

$$
V_{\gamma}(t, \varepsilon)=\left[\begin{array}{cc}
1-\frac{\varepsilon \lambda \gamma(t)}{\gamma(t)^{\prime}}, & \frac{\varepsilon \lambda \gamma(t)^{2}}{\gamma(t)^{\prime}} \\
-\frac{\varepsilon \lambda}{\gamma(t)^{\prime}}, & 1+\frac{\varepsilon \lambda \gamma(t)}{\gamma(t)^{\prime}}
\end{array}\right]
$$

This time-dependent Möbius transformation is the time- $\varepsilon$ flow of the vector field $\lambda \xi_{\gamma}$.

Then one has

$$
V_{\delta}(t,-\varepsilon) A_{\mu, \gamma, \delta}(t+\varepsilon) V_{\gamma}(t, \varepsilon)=V_{\delta}(t, \varepsilon) A_{\mu, \gamma, \delta}(t-\varepsilon) V_{\gamma}(t,-\varepsilon) \quad \bmod \varepsilon^{2},
$$


which is verified by a direct calculation or, in the limit $\varepsilon \rightarrow 0$,

$$
\left[\begin{array}{cc}
\frac{\delta(t)}{\delta^{\prime}(t)}, & \frac{\delta(t)^{2}}{\delta^{\prime}(t)} \\
-\frac{1}{\delta^{\prime}(t)}, & \frac{\delta(t)}{\delta^{\prime}(t)}
\end{array}\right] A_{\mu, \gamma, \delta}(t)-A_{\mu, \gamma, \delta}(t)\left[\begin{array}{cc}
\frac{\gamma(t)}{\gamma^{\prime}(t)}, & \frac{\gamma(t)^{2}}{\gamma^{\prime}(t)} \\
-\frac{1}{\gamma^{\prime}(t)}, & \frac{\gamma(t)}{\gamma^{\prime}(t)}
\end{array}\right]=\frac{1}{\lambda} A_{\mu, \gamma, \delta}^{\prime}(t) .
$$

This equality implies that the vector fields $\lambda \xi_{\gamma}$ and $\lambda \xi_{\delta}$ are conjugate, and so are $\Phi_{\lambda, \gamma}$ and $\Phi_{\lambda, \delta}$ :

$$
\Phi_{\lambda, \delta}=A_{\mu, \gamma, \delta}(0) \Phi_{\lambda, \gamma} A_{\mu, \gamma, \delta}^{-1}(0)
$$

as needed.

Remark 4.1 The above theorem is also a continuous analog of a result for ideal polygons in [1].

Bianchi permutability. Let us show that the maps $T_{c}$ commute; the argument is similar to that given in [1] for ideal polygons.

Theorem 5 Let three closed curves satisfy $\gamma \stackrel{c_{1}}{\sim} \gamma_{1}$ and $\gamma \stackrel{c_{2}}{\sim} \gamma_{2}$. Then there exists a fourth curve $\gamma_{12}$ such that $\gamma_{1} \stackrel{c_{2}}{\sim} \gamma_{12}$ and $\gamma_{2} \stackrel{c_{1}}{\sim} \gamma_{12}$.

Proof. We use (10), writing $A$ instead of $A(0)$.

Since $\gamma \stackrel{c_{1}}{\sim} \gamma_{1}$ and $\gamma \stackrel{c_{2}}{\sim} \gamma_{2}$, we have

$$
\Phi_{c_{1}, \gamma}\left(\gamma_{1}(0)\right)=\gamma_{1}(0), \Phi_{c_{2}, \gamma}\left(\gamma_{2}(0)\right)=\gamma_{2}(0)
$$

By (10),

$$
\Phi_{c_{1}, \gamma_{2}}=A_{\mu, \gamma, \gamma_{2}} \Phi_{c_{1}, \gamma} A_{\mu, \gamma, \gamma_{2}}^{-1}, \Phi_{c_{2}, \gamma_{1}}=A_{\nu, \gamma, \gamma_{1}} \Phi_{c_{2}, \gamma} A_{\nu, \gamma, \gamma_{1}}^{-1}
$$

with

$$
c_{1}=c_{2}(1-\mu), c_{2}=c_{1}(1-\nu)
$$

It follows that

$$
\Phi_{c_{1}, \gamma_{2}}\left(A_{\mu, \gamma, \gamma_{2}}\left(\gamma_{1}(0)\right)\right)=A_{\mu, \gamma, \gamma_{2}}\left(\gamma_{1}(0)\right), \Phi_{c_{2}, \gamma_{1}}\left(A_{\nu, \gamma, \gamma_{1}}\left(\gamma_{2}(0)\right)\right)=A_{\nu, \gamma, \gamma_{1}}\left(\gamma_{2}(0)\right)
$$

Thus we need to show that

$$
A_{\mu, \gamma, \gamma_{2}}\left(\gamma_{1}(0)\right)=A_{\nu, \gamma, \gamma_{1}}\left(\gamma_{2}(0)\right)
$$


This is indeed the case: (11) implies that $\frac{1}{\mu}+\frac{1}{\nu}=1$, and then a calculation shows that

$$
\frac{1}{\mu}\left[\begin{array}{cc}
\gamma-\mu \gamma_{2}, & \gamma \gamma_{2}(\mu-1) \\
1-\mu, & \gamma \mu-\gamma_{2}
\end{array}\right]\left[\begin{array}{c}
\gamma_{1} \\
1
\end{array}\right]=\frac{1}{\nu}\left[\begin{array}{cc}
\gamma-\nu \gamma_{1}, & \gamma \gamma_{1}(\nu-1) \\
1-\nu, & \gamma \nu-\gamma_{1}
\end{array}\right]\left[\begin{array}{c}
\gamma_{2} \\
1
\end{array}\right]
$$

as needed.

Remark 4.2 The above considerations can be extended to centro-affine twisted curves, that is, curves with monodromy, $\Gamma(t+\pi)=M(\Gamma(t))$, where the monodromy $M \in \mathrm{SL}(2, \mathbb{R})$ is not necessarily - Id. One can define the maps $T_{c}$ on twisted curves: given $\Gamma$, consider the respective $\pi$-periodic potential of the Hill equation $p(t)$, find a $\pi$-periodic solution $a(t)$ to equation (5), and define $\Delta=a \Gamma+c \Gamma^{\prime}$. Then the monodromy of $\Delta$ coincides with that of $\Gamma$. At the level of Hill's equations, this is the map $p \mapsto q$. We do not dwell on this extension here.

Acknowledgements. It is a pleasure to acknowledge the stimulating discussions with A. Calini, A. Izosimov, I. Izmestiev, B. Khesin, and V. Ovsienko. This work was supported by NSF grant DMS-1510055.

\section{References}

[1] M. Arnold, D. Fuchs, I. Izmestiev, S. Tabachnikov. Cross-ratio dynamics on ideal polygons. In preparation.

[2] A. Calini, T. Ivey, G. Marí-Beffa. Remarks on KdV-type flows on starshaped curves. Phys. D 238 (2009), 788-797.

[3] A. Fujioka, T. Kurose. Hamiltonian formalism for the higher KdV flows on the space of closed complex equicentroaffine curves. Int. J. Geom. Methods Mod. Phys. 7 (2010), 165-175.

[4] A. Fujioka, T. Kurose. Multi-Hamiltonian structures on spaces of closed equicentroaffine plane curves associated to higher KdV flows. SIGMA 10 (2014), Paper 048, 11 pp.

[5] E. L. Ince. Ordinary Differential Equations. Dover Publications, New York, 1944. 
[6] B. Khesin, R. Wendt. The geometry of infinite-dimensional groups. Springer-Verlag, Berlin, 2009.

[7] V. Ovsienko, S. Tabachnikov. Projective differential geometry old and new. From the Schwarzian derivative to the cohomology of diffeomorphism groups. Cambridge University Press, Cambridge, 2005.

[8] V. Ovsienko, S. Tabachnikov. Coxeter's frieze patterns and discretization of the Virasoro orbit. J. Geom. Phys. 87 (2015), 373-381.

[9] U. Pinkall. Hamiltonian flows on the space of star-shaped curves. Results Math. 27 (1995), 328-332.

[10] S. Tabachnikov. On the bicycle transformation and the filament equation: results and conjectures. J. Geom. Phys. 115 (2017), 116-123.

[11] C.-L. Terng, Z. Wu. Central affine curve flow on the plane. J. Fixed Point Theory Appl. 14 (2013), 375-396. 